\title{
Antagonistic Serotonergic and Octopaminergic Neural Circuits Mediate Food-Dependent Locomotory Behavior in Caenorhabditis elegans
}

\author{
Matthew A. Churgin, Richard J. McCloskey, Emily Peters, and Christopher Fang-Yen \\ Department of Bioengineering, School of Engineering and Applied Science, University of Pennsylvania, Philadelphia, Pennsylvania 19104-6321
}

\begin{abstract}
Biogenic amines are conserved signaling molecules that link food cues to behavior and metabolism in a wide variety of organisms. In the nematode Caenorhabditis elegans, the biogenic amines serotonin (5-HT) and octopamine regulate a number of food-related behaviors. Using a novel method for long-term quantitative behavioral imaging, we show that 5-HT and octopamine jointly influence locomotor activity and quiescence in feeding and fasting hermaphrodites, and we define the neural circuits through which this modulation occurs. We show that 5-HT produced by the ADF neurons acts via the SER-5 receptor in muscles and neurons to suppress quiescent behavior and promote roaming in fasting worms, whereas 5-HT produced by the NSM neurons acts on the MOD-1 receptor in AIY neurons to promote low-amplitude locomotor behavior characteristic of well fed animals. Octopamine, produced by the RIC neurons, acts via SER-3 and SER-6 receptors in SIA neurons to promote roaming behaviors characteristic of fasting animals. We find that 5-HT signaling is required for animals to assume food-appropriate behavior, whereas octopamine signaling is required for animals to assume fasting-appropriate behavior. The requirement for both neurotransmitters in both the feeding and fasting states enables increased behavioral adaptability. Our results define the molecular and neural pathways through which parallel biogenic amine signaling tunes behavior appropriately to nutrient conditions.
\end{abstract}

Key words: 5-HT; behavioral states; Caenorhabditis elegans; locomotion; octopamine; serotonin

Significance Statement

Animals adjust behavior in response to environmental changes, such as fluctuations in food abundance, to maximize survival and reproduction. Biogenic amines, such as like serotonin, are conserved neurotransmitters that regulate behavior and metabolism in relation to energy status. Disruptions of biogenic amine signaling contribute to human neurological diseases of mood, appetite, and movement. In this study, we investigated the roles of the biogenic amines serotonin and octopamine in regulating locomotion behaviors associated with feeding and fasting in the roundworm Caenorhabditis elegans. We identified neural circuits through which these signals work to govern behavior. Understanding the molecular pathways through which biogenic amines function in model organisms may improve our understanding of dysfunctions of appetite and behavior found in mammals, including humans.

\section{Introduction}

The ability to alter behavior in response to nutritional cues is a crucial trait for organisms to adapt to changing environments.

Received Aug. 18, 2016; revised May 18, 2017; accepted July 1, 2017.

Author contributions: M.A.C. and C.F.-Y. designed research; M.A.C., R.J.M., and E.P. performed research; M.A.C. analyzed data; M.A.C., R.J.M., and C.F.-Y. wrote the paper.

C.F.-Y. was supported by the National Institutes of Health (R01-NS-084835), the Ellison Medical Foundation, and the Alfred P. Sloan Research Foundation. M.A.C. was supported by the National Institutes of Health (R01-NS084835). R.J.M. was supported by the National Institutes of Health (T32 HL-007712-23). Some of the strains used in this study were provided by the Caenorhabditis Genetics Center, which is funded by the National Institutes of Health Office of Research Infrastructure Programs (P40 0D010440). We thank H. Robert Horvitz, Ji Ying Sze, Supriya Srinivasan, Zheng-Xing Wu, and Satoshi Suo for sharing strains and plasmid reagents. We thank David M. Raizen for helpful discussions, technical assistance, and sharing of laboratory resources.

The authors declare no competing financial interests.

Correspondence should be addressed to Christopher Fang-Yen at the above address. E-mail: fangyen@seas.upenn.edu.
For example, many animals respond to scarcity of food by adopting quiescent behaviors, such as hibernation or diapause (Nelson et al., 2014; van Breukelen and Martin, 2015). Hibernation is a long-term behavior characterized by suppressed movement and metabolism that occurs in response to low food availability and decreased temperature (Carey et al., 2003). How the nervous system initiates and sustains quiescent behavior during hibernation is poorly understood (Andrews, 2007).

In many organisms, the linking of behavior and metabolism (Roeder, 2005; Chase and Koelle, 2007; Tecott, 2007) to nutrition cues is performed in part through a widely conserved class of signaling molecules called biogenic amines. In mammals, bio- 
genic amines regulate appetite, mood, weight, and other aspects of physiology (Lam, 2006). Such disorders as anorexia, obesity, and depression, whose symptoms include altered food intake, are associated with dysregulation of the biogenic amine serotonin (5-HT; Curran and Chalasani, 2012). These disorders can be treated with drugs that increase the amount of 5-HT acting at the synapse, but how 5-HT levels relate to the complex behavioral and metabolic manifestations of these disorders is not fully understood. Thus, it is important to understand the mechanisms by which biogenic amines link environmental stimuli to behavior and metabolism.

The nematode Caenorhabditis elegans, because of its compact nervous system, manipulable genetics, and a host of food-related behaviors, is a powerful model organism to dissect the neural circuits linking food, behavior, and physiology (Hart, 2006; Sengupta, 2013). Its nervous system signals in part through the biogenic amines 5-HT, dopamine, tyramine, and octopamine. Here we focus on the behavioral effects of 5-HT and octopamine in relation to food levels.

5 -HT is involved in the modulation of multiple C. elegans behaviors in relation to food levels (Ségalat et al., 1995; Liang et al., 2006). During feeding, 5-HT is released and promotes locomotory slowing, thereby increasing the probability of the animal remaining on a food source (Sawin et al., 2000). In addition, 5-HT increases pharyngeal pumping (feeding) and egg-laying rates on food (Waggoner et al., 1998; Song et al., 2013). Mutants lacking tryptophan hydroxylase, the rate-limiting enzyme required for 5-HT synthesis, exhibit an increased propensity to developmentally arrest, indicating that lack of the positive food signal, 5-HT, discourages development into adulthood (Sze et al., 2000).

While 5-HT is associated with food-related behaviors, multiple lines of evidence suggest that octopamine is associated with starvation-related behaviors. In Drosophila melanogaster, octopamine signaling is required for the hyperactivity observed in starved animals (Yang et al., 2015). Hyperactivity is thought to reflect the drive of starving animals to locate a food source. In C. elegans, octopamine inhibits egg laying and feeding, and modulates response rate to aversive stimuli (Alkema et al., 2005; Wragg et al., 2007; Mills et al., 2012). However, octopamine's role in C. elegans locomotion has not been well studied, and how it acts to regulate fooddependent locomotion behavior is unknown.

Recently, our laboratory investigated the dynamics of behavioral states of worms in liquid. McCloskey et al. found that food, mechanical environment, insulin signals, protein kinase $G$ signaling, and neuropeptides regulate the incidence of three behavioral states: roaming, dwelling, and quiescence (Ghosh and Emmons, 2008; McCloskey et al., 2017). While roles for acetylcholine, TGF- $\beta$, and insulin signaling have been reported in regulating these behavioral states, specific neural circuits have not been fully elucidated (You et al., 2008; Gallagher et al., 2013).

In this study, we used longitudinal imaging, pharmacology, and genetic techniques to show that the biogenic amines 5-HT and octopamine regulate behavior during feeding and fasting in C. elegans. We then identified the neuronal classes responsible for releasing these neurotransmitters and the receptors on which they act. We show that 5-HT and octopamine play antagonistic roles in regulating nutrient-related locomotory states.

\section{Materials and Methods}

Strains. C. elegans was cultured as previously described (Brenner, 1974). All worms were hermaphrodites grown on standard agar plates with OP50 Escherichia coli until just before experiments. N2 Bristol was used as the wild-type reference strain. The following strains were used in this study: MT15434: tph-1(mg280) II; DA1814: ser-1(ok345) X; CX12800: ser-3(ad1774) I; AQ866: ser-4(ok512) III; RB2277: ser-5(ok3087) I, ser5(tm2654) I, ser-5(tm2647) I; KQ1048: ser-6(tm2146) IV; DA2100: ser7(tm1325) X; MT9668: mod-1(ok103) V; MT9180: mod-1(n3034) V; MT9667: mod-1(nr2043) V; MT13113: tdc-1(n3419) II; MT9455: tbh-1(n3247) X; CX13079: octr-1(ok371) X; DA2289: tph-1(mg280) II, kyEx947[pceh-2::tph1(+)::gfp punc-122::gfp(+)]; DA2290: tph-1(mg280) II, kyEx949[psrh-142:: tph-1(+)::gfp punc-122::gfp(+)]; SSR664: mod-1(ok103) V, [Pmod-1::mod-1]; SSR644: mod-1(ok103) V, [Pttx-3::mod-1]; SSR642: $\bmod -1$ (ok103) V, [Pgcy-36:: mod-1]; SSR665: mod-1(ok103) V, [Pflp-1:mod-1]; SSR662: mod-1(ok103) V, [Pflp-8::mod-1]; VN289: ser-6(tm2104), tzIs3 [cre::sfp, lin-15(+)]; VN441: ser6(tm2104), tzIs3, vnEx142[ceh-17::ser-6, lin-44::gfp]; YX179: ser-5(ok3087) I, [Pser-5::ser-5, Pmyo-2::mCherry]; YX180: ser-5(ok3087) I, [Pmyo-3::ser-5, Pmyo2::mCherry]; YX181:ser-3(ad1774) I, [Pceh-17::ser-3, Pmyo-2::mCherry]; YX184: ser-5(ok3087) I, [Psra-6::ser-5, Pmyo-2::mCherry]; YX185: ser-5(ok3087) I, [Punc-119::ser-5, Pmyo-2::mCherry].

WorMotel design and fabrication. We fabricated the WorMotel, a custom microplate device consisting of an array of individual wells that can be used to longitudinally image animals under solid or liquid media conditions (Churgin et al., 2017). For this purpose, we developed a 3Dprinting-based molding method (Shepherd et al., 2011). We designed a chip containing a rectangular array of either 48 or 240 rounded wells. Each well was $3 \mathrm{~mm}$ in diameter and $3 \mathrm{~mm}$ deep, with spacing from center to center of $4.5 \mathrm{~mm}$ (Fig. 1). Each well was surrounded by a 0.5-mm-wide and 3-mm-deep channel, which served as a moat. Designs of the WorMotel masters were created using Matlab. We printed a master corresponding to the negative of this shape with an Objet30 photopolymer 3D printer using the material VeroBlack. To mold the WorMotel devices, we mixed Dow Corning Sylgard 184 polydimethylsiloxane (PDMS) according to the manufacturer's instructions and poured 35 or $5 \mathrm{~g}$ of PDMS into the 240 -well or 48 -well masters, respectively. We then degassed the poured PDMS in a vacuum chamber for $1 \mathrm{~h}$. Devices were cured overnight at $40^{\circ} \mathrm{C}$ and then removed from molds using a spatula.

Liquid behavioral assays. Nine microliters of liquid NGM buffer (NGB), which consists of the same components as NGM but without agar or peptone (Stiernagle, 2006; McCloskey et al., 2017), was added to each well of the WorMotel just before adding worms. Young-adult animals were added manually to the liquid contained in each well of the WorMotel. Where indicated, the NGM was supplemented with either drugs or food. As a food source, we used E. coli DA837 (Davis et al., 1995), a streptomycin-resistant derivative of OP50 (Brenner, 1974). For experiments where food was added to wells, bacteria was added to NGM to a final $\mathrm{OD}_{600}$ of $\sim 1$.

For experiments with added exogenous biogenic amines, we added 5-HT hydrochloride, tyramine hydrochloride, or octopamine hydrochloride (Sigma-Aldrich) in the indicated concentrations to liquid NGM before filling the WorMotel. Multiple genotypes or conditions were assessed on the same WorMotel. Behavioral comparisons were made only between conditions that were assayed simultaneously on the same WorMotel.

PDMS devices were placed inside a 90-mm-diameter Petri dish. To maintain humidity inside the dishes, we used water-absorbing polyacrylate crystals. Sterile water was added to the crystals in a ratio of 150:1 (water/crystals) by weight. Approximately $5 \mathrm{~g}$ of hydrated crystals were added around the WorMotel. We placed lids on all dishes. To prevent accumulation of water condensation, lids were prepared by coating with a 30\% solution of Tween 20 (Sigma-Aldrich) in water, which was allowed to dry before use.

Finally, the filled WorMotel was placed upside down on the bottom of the Petri plate and the lid was added. The Petri plate was placed on a glass platform inside a custom imaging rig as previously described (Churgin and Fang-Yen, 2015). A digital camera (see Image acquisition) was placed beneath the glass platform and images were acquired every second until 60,000 images had been acquired, corresponding to $\sim 16.6 \mathrm{~h}$ of recording time (Churgin and Fang-Yen, 2015).

Image acquisition. Images were captured with an Imaging Source DMK 23GP031 camera $(2592 \times 1944$ pixels $)$ equipped with a Fujinon lens (HF12.5SA-1; focal length, $12.5 \mathrm{~mm}$ ). We used IC Capture imaging soft- 
a

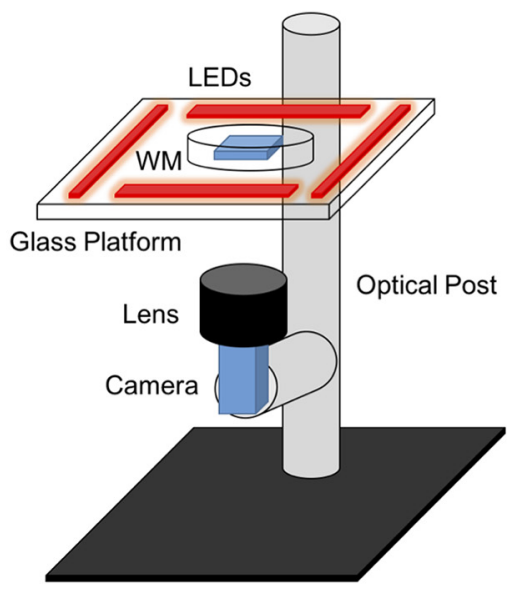

b

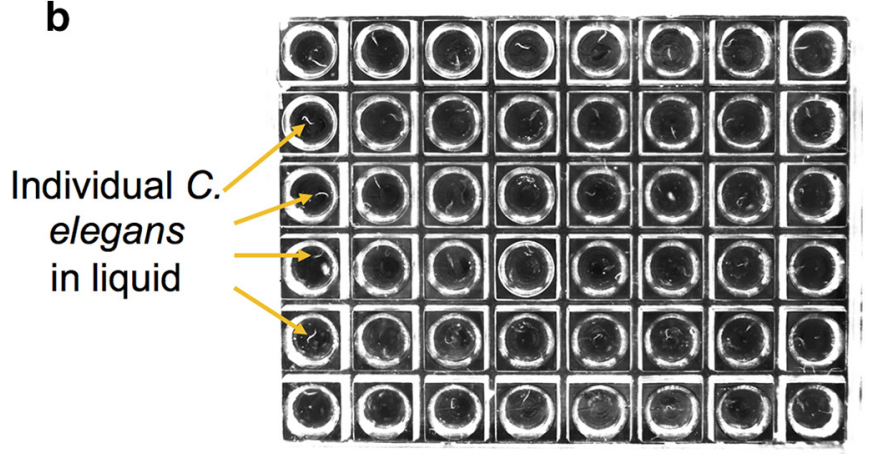

C

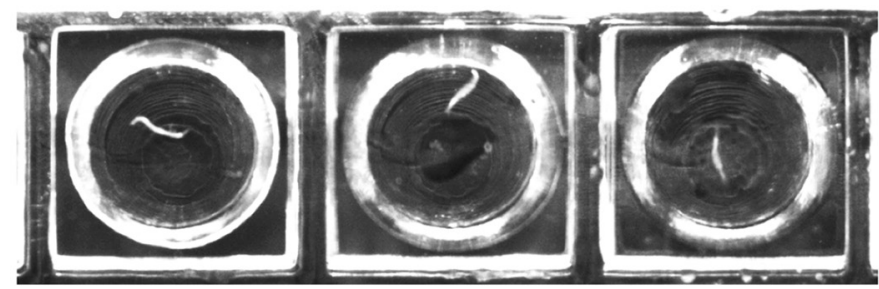

d

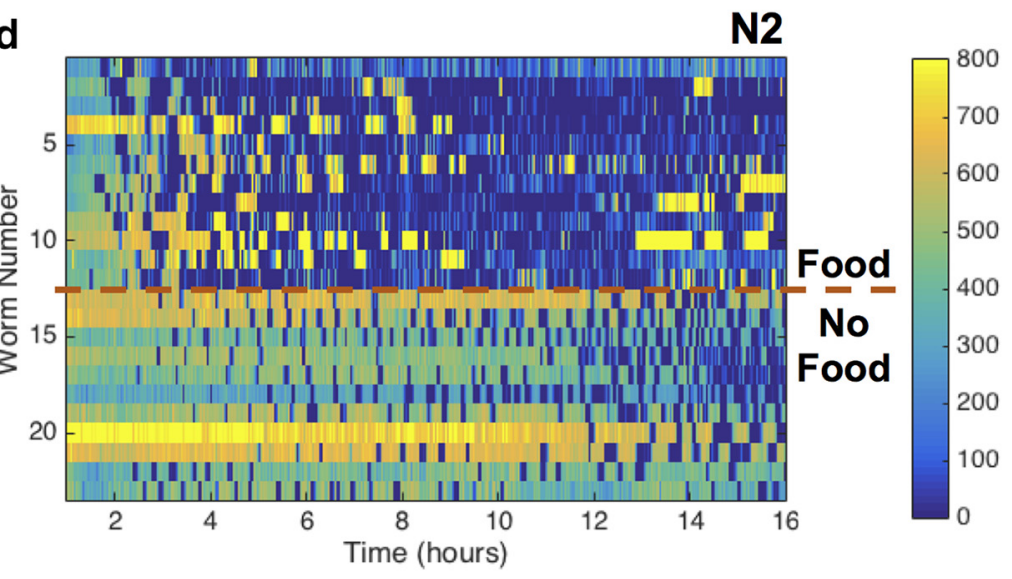

Figure 1. Experimental setup and data analysis. $\boldsymbol{a}$, Experimental setup. $\boldsymbol{b}$, Example image. Individual C. elegans are visible in each well. c, Detail of three wells. $\boldsymbol{d}$, Locomotion activity map for worms in liquid incubated with (top half) or without (bottom half) bacterial food.

ware (Imaging Source) to acquire time-lapse images through a gigabit Ethernet connection. All experiments were performed under dark-field illumination using four 4.7 inch red LED strips (Oznium) positioned on the glass platform surrounding the WorMotel. Images were saved and processed by a 64-bit computer with a 3.40 $\mathrm{GHz}$ Intel Core i3 processor and $4 \mathrm{~GB}$ of RAM. Images were processed and analyzed using custom-written Matlab software (see Image processing and data analysis).

The camera's field of view was adjusted such that 48 wells of the WorMotel were visible on the image. This field of view corresponded to a spatial resolution of $\sim 15 \mu \mathrm{m}$.

Image processing and data analysis. All data processing and analysis were performed using Matlab (Mathworks). Subsequent images were subtracted to generate difference images of pixel value intensity change. The difference image was normalized by average pixel intensity across the two subtracted images. A Gaussian smoothing filter with $\mathrm{SD}$ equal to one pixel was applied to the $\Delta$ image to reduce noise. A binary threshold of 0.35 was then applied to the filtered $\Delta$ image to score whether or not movement occurred at each pixel location. All pixels in which movement occurred were summed up and the resulting value was called the "activity" between the two frames.

To calculate the time spent quiescent, we first time-averaged raw activity data, with time resolution of $1 \mathrm{~s}$, with a smoothing kernel of $5 \mathrm{~s}$. For each animal, we then calculated the number of frames where the activity was equal to zero. The resultant value was divided by the duration of the assay to calculate the fraction of time spent quiescent.

Histogram fitting. We generated a histogram of the $5 \mathrm{~s}$ time-averaged activity for each worm. We then used a nonlinear least-squares fitting algorithm to fit each individual worm's activity histogram to the sum of two exponential terms (with two unknown parameters each) and a Gaussian term (with three unknown parameters). We therefore fit seven parameters. The zero bin on the activity histogram, corresponding to quiescent frames, was excluded from the histogram before the fitting process as the contribution of quiescence had already been calculated.

To calculate the fraction of time spent roaming, we calculated the area under the Gaussian curve component of the fit. To calculate the fraction of time spent dwelling, we calculated the area under the two exponential curve components of the fit.

Manual validation of behavior. We picked at random three fasting and three feeding worms from a previously recorded experiment to manually score roaming and dwelling behavior. We examined videos recorded at eight frames per second rather than one frame per second because we found it easier for a manual user to score behavior when data were recorded at a higher frame rate. Nevertheless, activity values were still calculated using frames captured $1 \mathrm{~s}$ apart as in all other experiments.

We examined behavior for $\sim 10 \mathrm{~min}$ at each of three time points: 2,3 , and $4.5 \mathrm{~h}$ from the start of the experiment. The purpose of using three time points was not to assess temporal variations in behavior, but to ensure all three types of behavior were observed for each worm. Indeed, all three behaviors including roaming, dwelling, and quiescence were observed for each worm manually scored. 
a

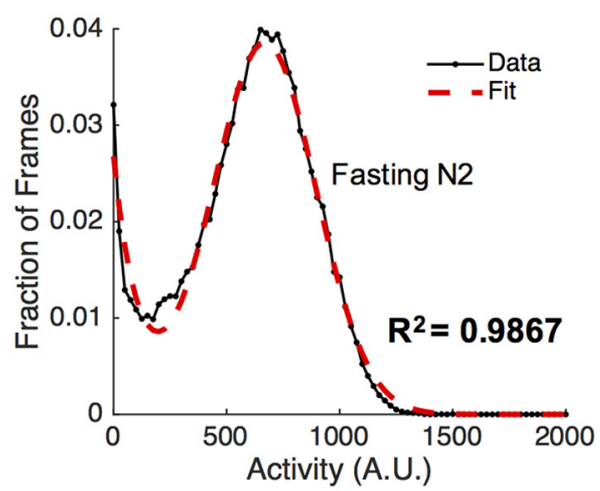

C

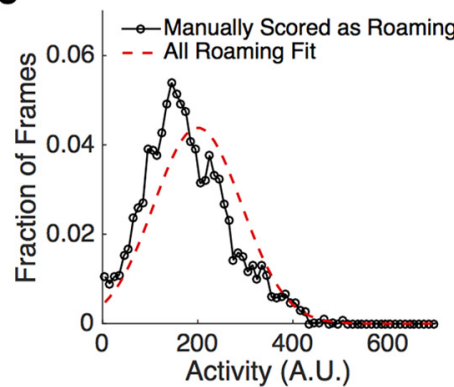

b

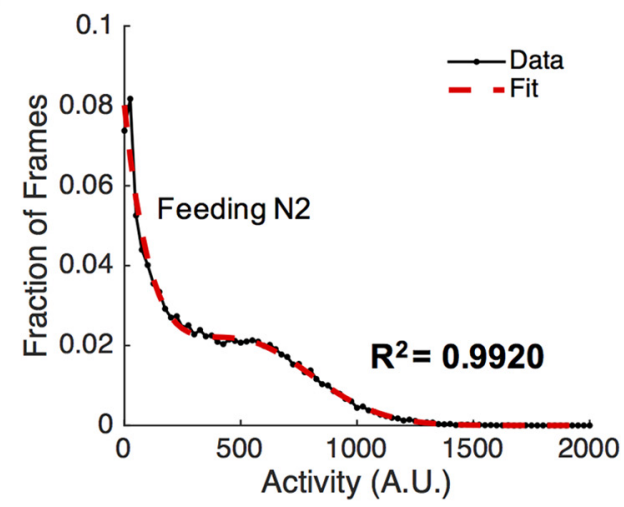

e

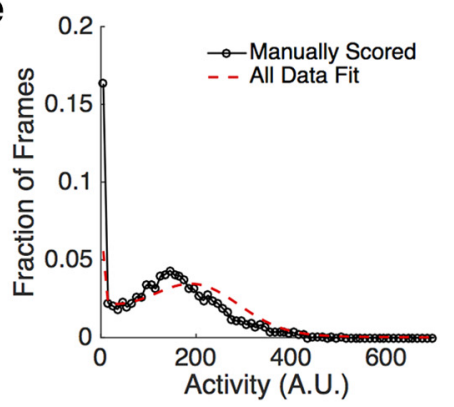

Figure 2. Quantitative analysis and manual validation. $\boldsymbol{a}$, Activity histogram for a fasting worm (black curve) with fit overlaid (red dashed curve). $\boldsymbol{b}$, Activity histogram for a feeding worm with fit overlaid. c, Activity histogram for frames during which a single worm's behavior was manually scored as roaming (black curve). The Gaussian component of the fit is overlaid (red dashed curve). $\boldsymbol{d}$, Activity histogram for frames during which the same worm's behavior as in c was manually scored as dwelling (black curve). The exponential component of the fit is overlaid (red dashed curve).e, Activity histogram for frames in the same worm's behavior as in c and $\boldsymbol{d}$ was manually scored. The full fit, consisting of both exponential and Gaussian components, is overlaid (red dashed curve).

A user blind to the results of the fit analysis scored behavior as roaming, dwelling, or quiescent for each worm for each frame.

Next, the activity histogram for frames the user scored as roaming and dwelling were individually generated and compared with the Gaussian or exponential components, respectively, of the full-data fit. To quantify the agreement between user-scored and computer-scored behavior, we calculated the $R^{2}$ value.

Visualizing data in roaming-dwelling space. After fitting, the roaming and dwelling fraction was calculated for each worm of a given genotype and experimental condition. For each population, the mean roaming and dwelling fraction was calculated, along with the SD and orientation of principal components in roaming-dwelling space. Ellipses with dimensions corresponding to the SEM for each experimental condition were plotted with their orientation in the direction of greatest variation for that experimental condition as determined by principal component analysis.

Cloning and transgenic strain construction. Rescue constructs for SER-5 were cloned using Gateway Technology (Life Technologies). The desired promoters, ser-5 cDNA, and unc-54 3' UTR were acquired in entry slots 1,2 , and 3 , respectively. The following plasmids in Gateway entry vectors were gifts of Zheng-Xing Wu: sra-6p, ser-5p, and ser-5 cDNA (Guo et al., 2015). The LR recombination reaction was performed to produce each rescue construct. Each rescue plasmid was confirmed via sequencing. The rescue plasmid ceh-17::ser-3 was a gift from Satoshi Suo (Yoshida et al., 2014). Transgenic strains were constructed by microinjection in the C. elegans germline. SER-5 rescue constructs were injected into RB2277: ser-5(ok3087) mutants. SER-3 rescue constructs were injected into CX12800: ser-3(ad1774). For microinjections, $30 \mathrm{ng} / \mu \mathrm{l}$ of the desired plasmid was injected with $2 \mathrm{ng} / \mu \mathrm{l}$ of myo-2::mCherry coinjection marker and $100 \mathrm{ng} / \mu \mathrm{l}$ of $1 \mathrm{~kb}$ DNA ladder.

Experimental design and statistical analysis. Differences in population behavior distributions were performed using a two-tailed $t$ test. Error bars throughout the manuscript are presented as SEM, with $N$ taken to be the number of biological replicates per condition.
Table 1. Manual behavior score versus automatic fit $R^{2}$ values

\begin{tabular}{llll}
\hline $\begin{array}{l}\text { Manual score versus } \\
\text { automatic fit } R^{2} \text { (mean } \pm \text { SD) }\end{array}$ & Worm \#1 & Worm \#2 & Worm \#3 \\
\hline Feeding, roaming $R^{2}=0.86 \pm 0.07$ & $R^{2}=0.86$ & $R^{2}=0.94$ & $R^{2}=0.78$ \\
Feeding, dwelling $R^{2}=0.77 \pm 0.13$ & $R^{2}=0.62$ & $R^{2}=0.85$ & $R^{2}=0.84$ \\
Fasting, roaming $R^{2}=0.76 \pm 0.01$ & $R^{2}=0.74$ & $R^{2}=0.77$ & $R^{2}=0.76$ \\
Fasting, dwelling $R^{2}=0.58 \pm 0.19$ & $R^{2}=0.76$ & $R^{2}=0.38$ & $R^{2}=0.59$ \\
\hline
\end{tabular}

\section{Results}

\section{Quantification of locomotion behavior in swimming}

\section{C. elegans with longitudinal imaging}

We first sought to better characterize C. elegans behavior in feeding and fasting conditions. We monitored locomotion behavior of youngadult worms using the WorMotel (Churgin et al., 2017). We assayed worms under liquid conditions and quantified locomotion behavior using custom machine vision software (see Materials and Methods).

We first monitored young-adult N2 worms for $16 \mathrm{~h}$ in liquid NGB (see Materials and Methods) grown with or without food (Fig. 1). As previously reported, after a period of a few hours of continuous high activity, fasting worms in liquid began to cycle between high activity and behavioral quiescence (Fig. 1) (Ghosh and Emmons, 2008; McCloskey et al., 2017). The behavior of feeding and fasting worms was qualitatively very different. Fasting worms oscillated between extended high-activity periods and short low-activity periods, whereas feeding worms exhibited much more intermediate activity. We then plotted activity histograms for individual worms. From these histograms, we confirmed that fasting worms exhibit a more bimodal activity distribution, whereas feeding worms exhibit a mostly monotonically decreasing activity distribution. 

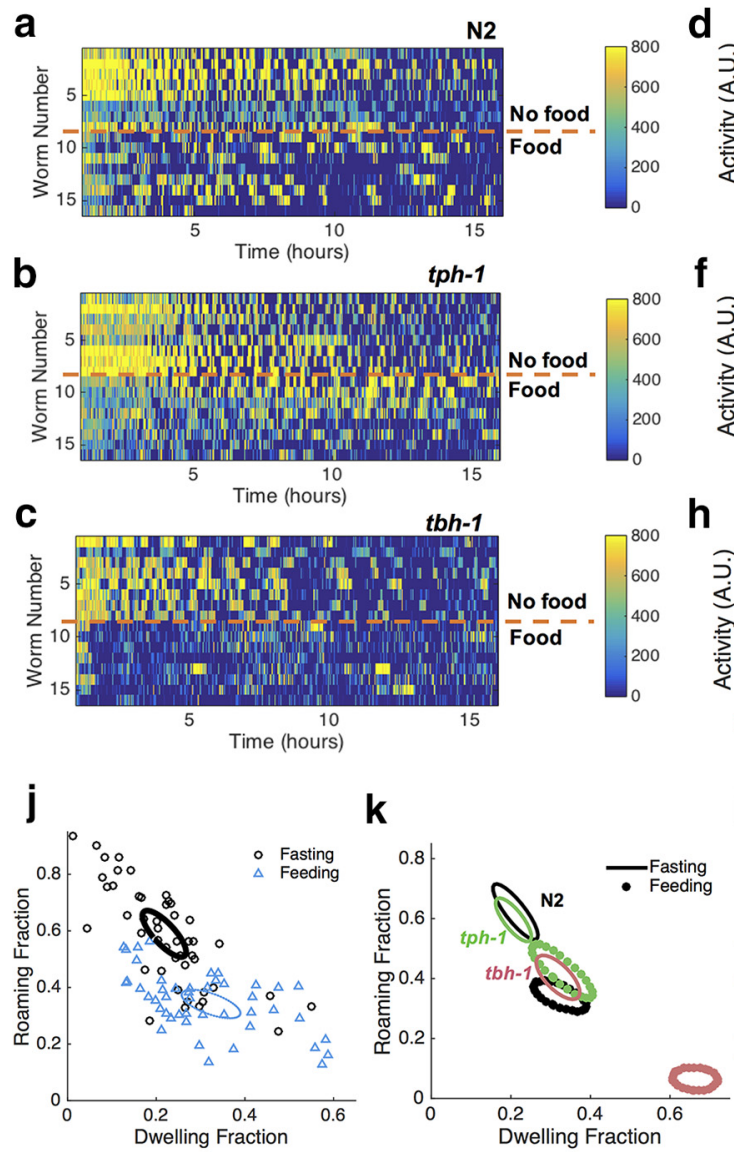
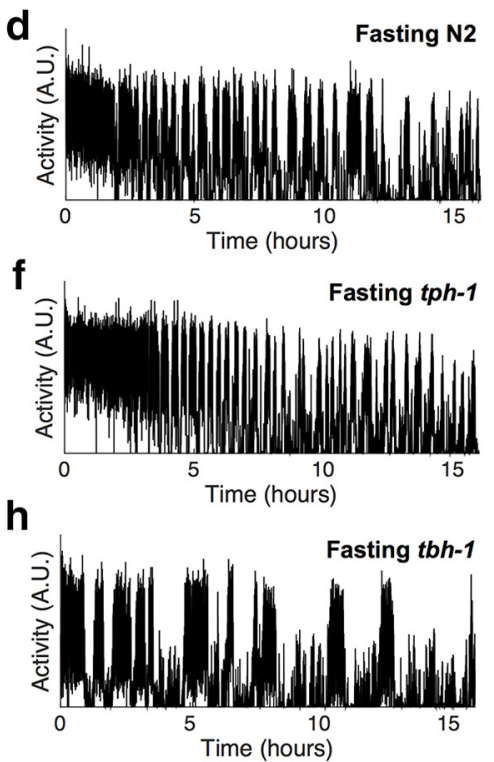

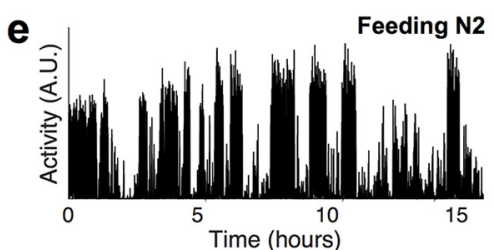

g
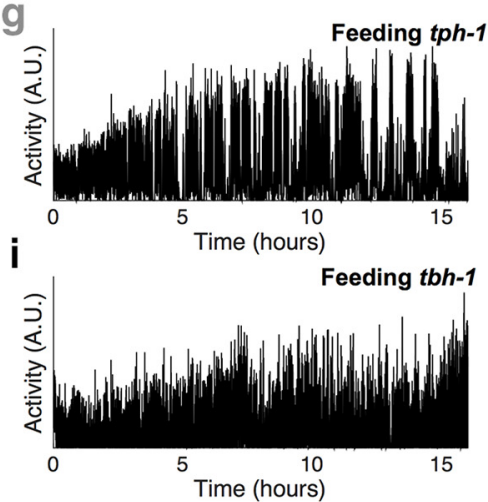

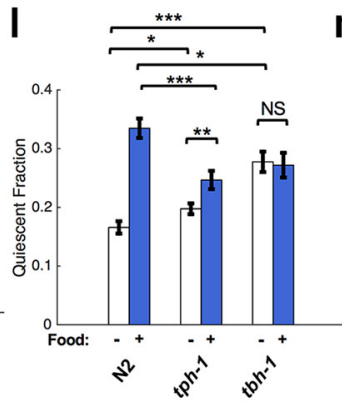

m

n

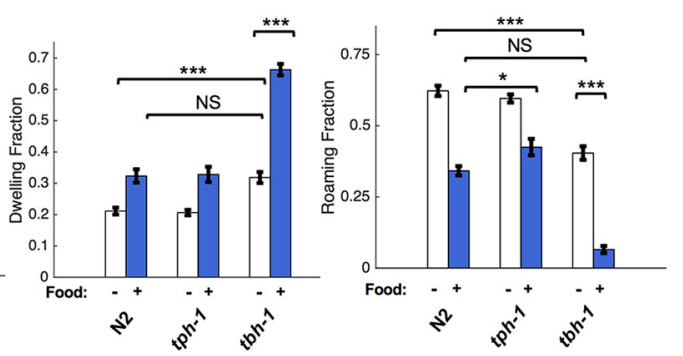

Figure 3. Serotonin and octopamine signaling are required for normal fasting and feeding behavior. $\boldsymbol{a}-\boldsymbol{c}$, Locomotion activity maps for N2, tph-1, and tbh-1, respectively. $\boldsymbol{d}$, Example activity trace for a fasting N2 worm. $\boldsymbol{e}$, Example activity trace for a feeding N2 worm. $\boldsymbol{f}$, Example activity trace for a fasting tph-1 worm. $\boldsymbol{g}$, Example activity trace for a feeding tph-1 worm. $\boldsymbol{h}$, Example activity trace for a fasting thh- 1 worm. $\boldsymbol{i}$, Example activity trace for a feeding tbh- 7 worm. $\boldsymbol{j}$, Roaming fraction versus dwelling fraction for individual fasting (black circles) or feeding (blue triangles) N2 worms. Principal component ellipses are plotted to indicate population averages and SEs. $\boldsymbol{k}$, Principal component ellipses illustrating fraction of time spent roaming versus dwelling for N2 (black), tph-1 (green), and tbh-1 (red) under fasting (solid ellipses) or feeding (dotted ellipses) conditions. Ellipse dimensions correspond to SEM. I, Quantification of fraction of time spent quiescent for N2 ( $n=$ 132), tph-1 $(n=132)$, and tbh-1 $(n=48)$ worms under fasting and feeding conditions. $\boldsymbol{m}$, Fraction of time spent dwelling. $n$, Fraction of time spent roaming. ${ }^{*} p<0.05 ;{ }^{* *} p<0.01 ;{ }^{* * *} p<0.005$.

To quantify behavior, we applied an empirical fit to the activity histogram on an individual worm basis (Fig. $2 a, b)$. We used a nonlinear least-squares algorithm to fit each worm's activity histogram to a sum of two exponential terms and one Gaussian term (see Materials and Methods). Worm locomotion is usually classified into one of three behavior states: roaming, dwelling, or quiescence (Flavell et al., 2013; McCloskey et al., 2017). Roaming is characterized by rapid movement and propagation of body waves down the length of the worm's body. Dwelling is characterized by less movement and the propagation of body waves in the anterior but not posterior of the worm's body. Quiescence is characterized by complete cessation of movement and is observed both in ample food environments, where it is termed satiety, and in fasting conditions, where it is termed fasting quiescence (Ghosh and Emmons, 2008; You et al., 2008). We therefore wanted to classify worm activity measurements according to these commonly applied labels.

We hypothesized that the exponential and Gaussian terms of the fit corresponded to dwelling and roaming behavior, respectively. To test this idea, we performed manual assessment of behavioral states from recorded videos. We found a strong overlap between the Gaussian component of the fit with the histogram of frames in which a worm's behavior was manually scored as roaming (Fig. 2c). Similarly, we found a strong overlap between the exponential components of the fit and the histogram of frames in which a worm's behavior was manually scored as dwelling (Fig. $2 d$ ). Combining both frames scored as dwelling and roaming together largely matched the overall fit (Fig. 2e; Table 1).

These results indicate that the exponential components of the histogram fit correspond well with frames in which worms are dwelling, and the Gaussian component corresponds with frames in which worms are roaming. Therefore, for each worm we simply summed up the area under each of these two components to calculate the fraction of time individuals spent dwelling and roaming. Quiescence was calculated as the fraction of time each worm's activity level was zero.

We found large quantitative differences in locomotion behavior between feeding and starved worms. We found that feeding caused an elevation in behavioral quiescence, a reduction in roaming, and an increase in dwelling compared with fasting (Fig. $3 j-n)$. Therefore, food status modulates multiple locomotion parameters in young-adult worms.

\section{5-HT signaling is required for worms to appropriately adapt} behavior from fasting to feeding

Having established that food levels affect locomotion behaviors, we sought to determine which neurotransmitters were responsible for these changes. Since serotonin is a transmitter known to regulate 
behavior in response to food, we first examined the behavior of $t p h-1$ mutants, which lack endogenous 5-HT, in response to feeding and fasting. These mutants exhibit increased fasting quiescence, decreased feeding quiescence, and increased roaming while feeding (Fig. 3). Therefore, endogenous 5-HT signaling is required for both wild-type fasting and feeding behavior.

We found that $t p h-1$ worms exhibited more roaming compared with N2 worms when assayed with food (Fig. 3). This is consistent with previous reports that $t p h-1$ worms roam more on a bacterial lawn than N2 worms (Flavell et al., 2013). Moreover, we found that food treatment in tph-1 animals had a lesser effect on the fraction of time spent quiescent. These results indicate that $t p h-1$ mutants are unable to fully adopt wild-type feeding behaviors in response to food. Our findings are consistent with the idea that $5-\mathrm{HT}$ is released in response to food and is required for adapting locomotion behaviors to a feeding-appropriate state.

\section{Differential effects of 5-HT produced by the ADF and NSM neurons}

We asked in what neurons 5-HT is produced to regulate locomotory fasting quiescence. 5-HT is produced primarily by two neuron pairs in the head, ADFL/ ADFR and NSML/NSMR, and one neuron pair near the vulva, HSNL/HSNR, as determined by expression of a Ptph-1::GFP transgene as well as antibody staining (Sze et al., 2002; Zheng et al., 2005; Liang et al., 2006). Previous reports showed that either fasting (Cunningham et al., 2012) or daf-2 mutation (Estevez et al., 2006) caused a reduction in tph-1 reporter expression only in the ADF neuron, implying that 5 -HT production by ADF is reduced in response to a reduction in nutrient signals.

We hypothesized that 5-HT in the ADF neurons plays a role in fasting quiescence. To test this idea, we examined tph-1 mutant worms in which wild-type TPH-1 was rescued in the ADF or NSM neurons with the cell-specific promoters srh-142 or ceh-2, respectively (Song et al., 2013). As before, we noticed that $t p h-1$ mutants exhibit less roaming and less dwelling compared with wild type (Fig. $4 a$ ). We found that $t p h-1$ rescue in ADF, but not in NSM, was sufficient to rescue roaming and dwelling to wild-type levels, whereas rescue of $t p h-1$ in NSM caused a drastic reduction in roaming (Fig. 4a). Therefore, these data suggest that 5-HT produced by the ADF neuron is required for wild-type fasting behavior, whereas 5-HT produced by the NSM neuron antagonizes wild-type fasting behavior.

As before, $t p h-1$ mutants exhibited slightly but significantly more quiescence than wild type, and this increase was restored to wild-type levels by rescue of TPH-1 in ADF (Fig. 4b). By contrast, rescue of TPH-1 in NSM resulted in a large increase in the fraction of time spent quiescent (Fig. $4 b$ ). As before, fasting tph-1 mutants exhibited an insignificant reduction in roaming, and this reduction was reversed by rescue of TPH-1 in ADF (Fig. 4a). By contrast, rescue of TPH-1 in NSM exacerbated the reduction of roaming, suggesting that these two neurons act antagonistically to regulate the amount of roaming.
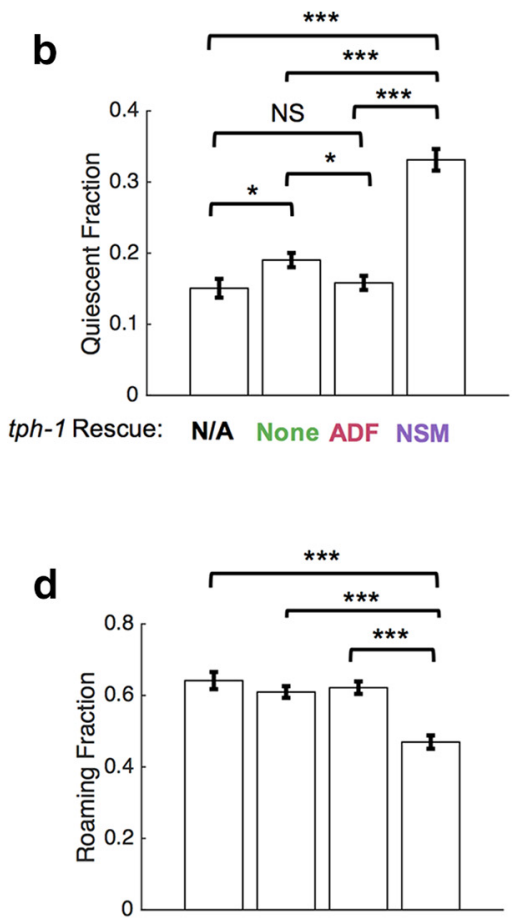

tph-1 Rescue: N/A None ADF NSM

Figure 4. Antagonistic roles for two serotonergic neurons. $\boldsymbol{a}$, Principal component ellipses illustrating fraction of time spent s) $\boldsymbol{b}$, Fraction of time spent quiescent. $\boldsymbol{c}$, Fraction of time spent dwelling $\boldsymbol{d}$, Fraction of time spent roaming. In all graphs, genotypes represented are N2 $(n=84)$, tph-1 $(m g 280)(n=84)$, tph-1; Ex[Psrh-142::tph-1 $(+)](n=84)$, and tph-1; Ex[Pceh$2:: t p h-1(+)](n=80) .{ }^{*} p<0.05 ;{ }^{* *} p<0.01 ;{ }^{* * *} p<0.005$.

These results show that 5 -HT produced by the ADF neurons is necessary and sufficient to restore wild-type fasting quiescence and behavior. Furthermore, these findings show that ADF and NSM act antagonistically with respect to roaming behavior.

\section{Exogenous 5-HT suppresses fasting quiescence and increases dwelling}

Having established that endogenous 5-HT signaling regulates fasting quiescence, we sought to determine the effect of exogenous 5-HT on the behavior of fasting worms. When we added exogenous 5-HT to the liquid media, we observed in wild-type worms an increase in dwelling and reduction in roaming (Fig. $5 a, b$ ). Consistent with our results that $t p h-1$ mutants lacking 5-HT signaling exhibit higher fasting quiescence (Figs. 3, 4), treatment with exogenous 5-HT suppressed wild-type fasting quiescence (Fig. $5 c$ ).

Although exogenous 5-HT suppressed quiescence, it also reduced roaming (Fig. 5e). Therefore, exogenous 5-HT both reduces overall movement while also reducing the time spent completely immobile. Accordingly, exogenous 5-HT causes a dramatic increase in dwelling (Fig. $5 d$ ). These results are consistent with reports that 5-HT slows locomotion (Ranganathan et al., 2000; Flavell et al., 2013). Therefore, exogenous 5-HT acts to promote dwelling behavior and suppress both quiescence and roaming.

\section{5-HT promotes roaming via SER-5 and dwelling via MOD-1}

We sought to determine the receptors through which exogenous 5-HT modulates locomotion. In C. elegans, 5-HT acts strongly through four G-protein-coupled receptors and one 5-HT-gated chloride channel to regulate locomotion, feeding, fat storage, egg laying, and other phenotypes (Ranganathan et al., 2000; CarrePierrat et al., 2006; Gürel et al., 2012). To determine the receptors 

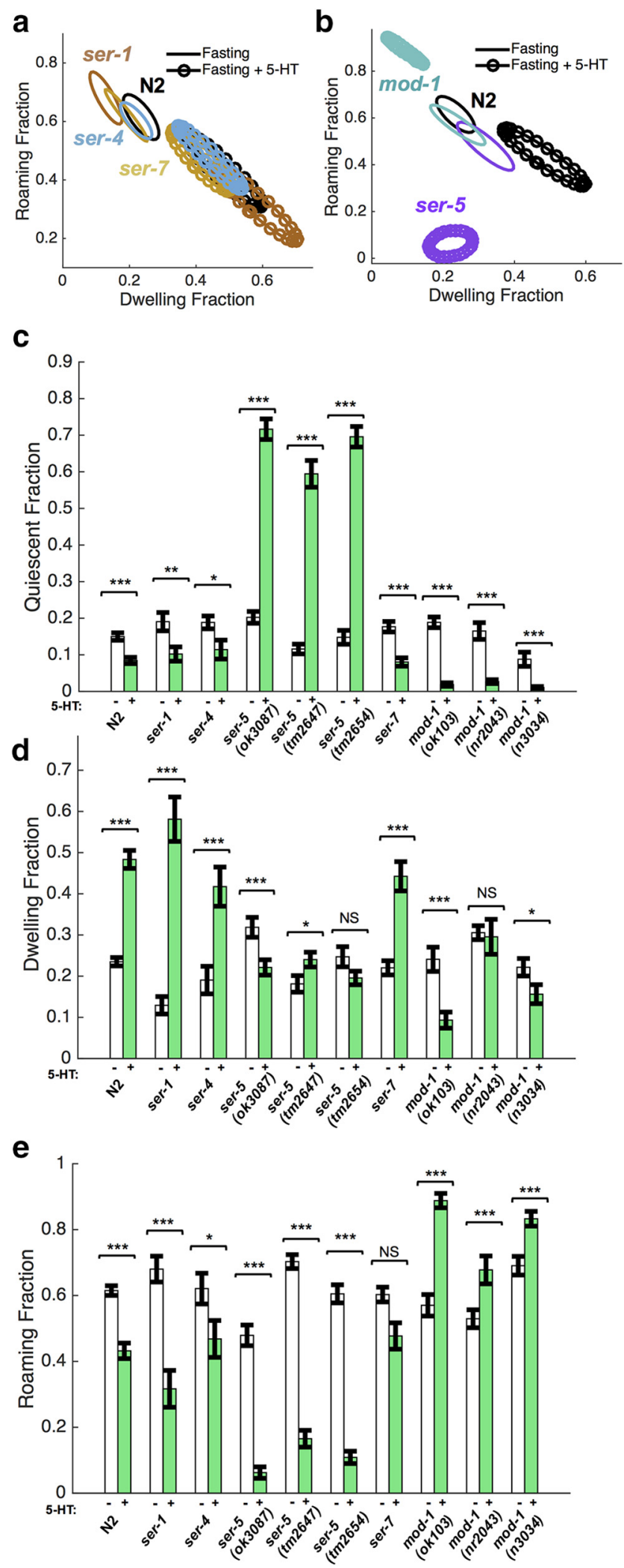

Figure 5. Exogenous serotonin acts via SER-5 to promote roaming and through MOD-1 to suppress roaming. $\boldsymbol{a}$, Principal component ellipses illustrating fraction of time spent roaming versus dwelling for N2 (black), ser-1 (brown), ser-4 (blue), and ser-7 (yellow) under fasting (solid ellipses) or fasting $+10 \mathrm{~mm} 5$-HT (dotted ellipses) conditions. $\boldsymbol{b}$, Principal component ellipses illustrating fraction of time spent roaming versus dwelling for N2 (black), ser-5 (purple), and mod-1 (cyan) under fasting or fasting $+10 \mathrm{~mm} 5-\mathrm{HT}$ conditions. c, Fraction of time spent quiescent. Number of animals tested were equal for both with and without 5 -HT conditions: N2 ( $n=124)$, ser-1 $(n=26)$, ser-4 $(n=18)$, ser-5(ok3087) $(n=54)$, ser-5 (tm2647) $(n=24)$, on which 5-HT acts, we tested under fasting conditions the available single 5-HT receptor mutants with and without exogenous 5-HT.

We found that exogenous 5-HT treatment had similar effects on quiescence, roaming, and dwelling in wild-type animals as in ser-1, ser-4, and ser- 7 mutants (Fig. $5 a$ ), indicating that these receptors do not contribute significantly to locomotion behavior. By contrast, exogenous 5-HT had a drastic effect on behavior of both ser-5 and mod-1 mutants (Fig. 5b). We found that 5-HT suppressed quiescence in all 5-HT receptor mutants tested except for ser-5 (Fig. 5c). Instead of suppressing quiescence in ser-5 mutants, applying exogenous 5-HT resulted in greatly increased quiescence in three different alleles compared with untreated worms. Furthermore, roaming was strongly suppressed in 5-HT-treated ser-5 mutants, indicating that SER- 5 promotes movement in response to 5 -HT.

Applying exogenous 5-HT to mod-1 mutants, by contrast, resulted in quiescence suppression in three different alleles to a greater degree than for wild-type worms (Fig. $5 c$ ). Furthermore, roaming increased and dwelling decreased in 5-HT-treated mod-1 mutants (Fig. $5 d, e$ ), indicating that $5-\mathrm{HT}$ acts via MOD-1 to suppress movement. These results are consistent with reports showing that MOD-1 promotes dwelling behavior (Ranganathan et al., 2000; Flavell et al., 2013). Together, our results indicate that 5-HT acts antagonistically through SER-5 to increase roaming and suppress quiescence and through MOD-1 to increase dwelling and suppress roaming (see Fig. 10).

MOD-1 acts in AIY neurons and SER-5 acts in body wall muscles and neurons to mediate behavioral effects of 5-HT

We next sought to determine in what cells or tissues the MOD-1 and SER-5 receptors act to mediate 5-HT's effects on behavior. MOD-1 is a 5-HT-gated chloride channel (Ranganathan et al., 2000) that has been shown to act in the nervous system to mediate locomotion (Flavell et al., 2013), learning (Zhang et al., 2005), and fat levels (Noble et al., 2013). SER-5 is a G-protein-coupled receptor that has been shown to act in the ASH neurons to mediate the behavioral response to octanol and in muscles to mediate egg laying (Hapiak et al., 2009; Harris et al., 2009). We therefore performed rescue experiments for MOD-1 and SER-5 in neurons, muscle, and specific cells to identify where these receptors act to mediate behavioral responses to 5-HT.

We first tested strains with a mod-1 mutant background in which wild-type MOD-1 was expressed under its endogenous promoter as well as under four neuron-specific promoters: Pttx-3, which is expressed in AIY, AIA, and AIZ neurons; Pflp-8, which is expressed in AUA and URX neurons; Pflp-1, which is expressed in RIG, AIA, and AIY neurons; and Pgcy-36, which is expressed in URX neurons (Noble et al., 2013). We found that rescuing MOD-1 under its endogenous promoter as well under the TTX-3 promoter resulted in partial rescue of wild-type behavior when treated with 5-HT (Fig. 6a-e). By contrast, rescuing MOD-1 under the FLP-1, FLP-8, or GCY-36 promoters did not result in behavior distinguishable from mod-1 mutants (Fig. $6 a-e$ ). The TTX-3 promoter has been previously reported to intensely drive expression in the AIY interneurons (Flavell et al., 2013). Therefore, our results

ser-5(tm2654) $(n=24), \operatorname{ser}-7(n=38), \bmod -1(0 k 103)(n=28), \bmod -1(n r 2043)(n=24)$, mod-1 $(n 3034)(n=16)$. d, Fraction of time spent dwelling.e, Fraction of time spent roaming. ${ }^{*} p<0.05 ;{ }^{* *} p<0.01 ;{ }^{* * *} p<0.005$. 

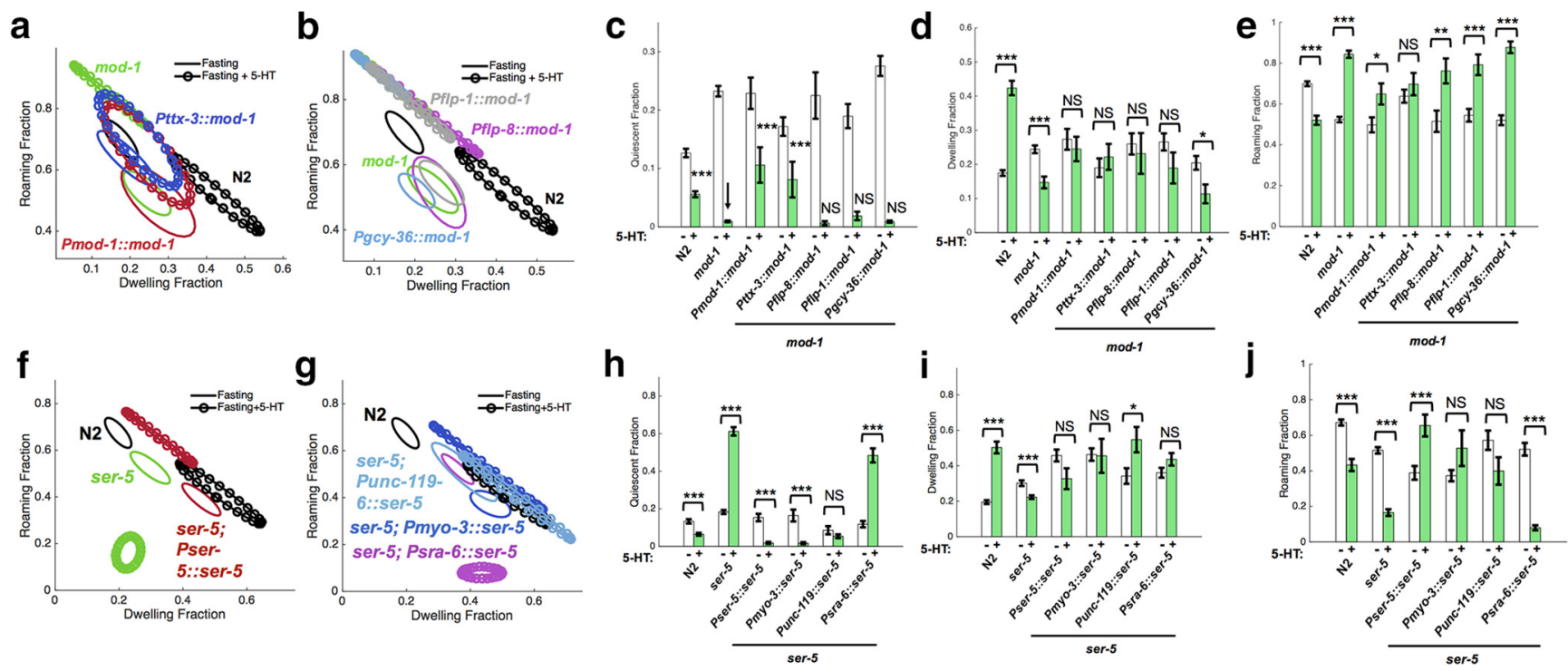

Figure 6. MOD-1 acts in AIY neurons and SER-5 acts in muscle and neurons to mediate 5-HT's effects on locomotor behavior. $\boldsymbol{a}$, Principal component ellipses illustrating fraction of time spent roaming versus dwelling for N2 (black), mod-1 (green), mod-1;Pmod-1::mod-1 (red), and mod-1;:Pttx-3::mod-1 (blue) under fasting (solid ellipses) or fasting +10 mm 5-HT (dotted ellipses) conditions. $\boldsymbol{b}$, Principal component ellipses illustrating fraction of time spent roaming versus dwelling for N2 (black), mod-1 (green), mod-1;:Pflp-1::mod-1 (gray), mod-1;Pflp-8::mod-1 (magenta), and mod-1;Pgcy-36::mod-1 (blue) under fasting or fasting $+10 \mathrm{~mm} 5$-HT conditions. C, Fraction of time spent quiescent. Number of animals tested were equal for both with and without 5-HT conditions: N2 ( $n=148), \bmod -1(n=148), \bmod -1 ;$ Pmod-1::mod-1 $(n=50), \bmod -1 ; P t t x-3:: \bmod -1(n=38), \bmod -1 ;$ Pflp-8::mod-1 $(n=22), \bmod -1 ; P f l p-1:: \bmod -1$ ( $n=24), \bmod -1 ;$ Pgcy36::mod-1 $(n=24)$. Significance markings are relative to mod-1 worms treated with 5 -HT (arrow). $\boldsymbol{d}$, Fraction of time spent dwelling. $\boldsymbol{e}$, Fraction of time spent roaming. $\boldsymbol{f}$, Principal component ellipses illustrating fraction of time spent roaming versus dwelling for N2 (black), ser-5 (green), ser-5;:Pser-5::ser-5 (red) under fasting (solid ellipses) or fasting + $10 \mathrm{~mm} 5-\mathrm{HT}$ (dotted ellipses) conditions. g, Principal component ellipses illustrating fraction of time spent roaming versus dwelling for N2 (black), ser-5 (green), ser-5;:Pmyo-3::ser-5 (blue), ser-5;Punc- $119::$ ser-5 (light blue), and ser-5; Psra-6::ser-5 (magenta) under fasting (solid ellipses) or fasting $+10 \mathrm{~mm}$ 5-HT (dotted ellipses) conditions. $\boldsymbol{h}$, Fraction of time spent quiescent. Number of animals tested were equal for both with and without 5-HT conditions: N2 ( $n=72)$, ser-5 $(n=72)$, ser-5;Pser-5::ser-5 $(n=16)$, ser-5;Pmyo-3::ser-5 $(n=16)$, ser-5;Punc-119::mod-1 $(n=24)$, ser-5;Psra-6::mod-1 $(n=16) . i$, Fraction of time spent dwelling. $j$, Fraction of time spent roaming. ${ }^{*} p<0.05 ;{ }^{* * *} p<0.005$.

show that MOD-1 expressed in the AIY neurons is necessary and sufficient to restore wild-type response to 5-HT.

Next, we generated rescue constructs for the SER- 5 receptor under its endogenous promoter, a neuron-specific promoter (Punc-119), a body wall muscle-specific promoter (Pmyo-3), and an ASH-specific promoter (Psra-6; see Materials and Methods), and injected them into a ser-5 mutant background. We found that rescuing SER-5 under its endogenous promoter fully restored the wild-type response to 5-HT (Fig. $6 f-j$ ). We found that rescuing SER-5 in either muscles or neurons rescued the behavioral response to 5-HT, whereas rescuing SER-5 in only the ASH neuron did not (Fig. $6 f-j$ ). Therefore, SER- 5 can act in both muscle or neurons to promote movement in response to 5-HT.

\section{Octopamine and tyramine have opposite effects on locomotion}

We next turned our attention toward neurotransmitters typically associated with fasting. The biogenic amines tyramine and octopamine modulate multiple behaviors and appear to signal starvation and promote starvation-related behaviors, such as suppressed pumping and egg laying (Rex and Komuniecki, 2002; Rex et al., 2004; Alkema et al., 2005; Suo et al., 2006). We sought to determine the roles, if any, of these neurotransmitters in locomotion behavior mediated in relation to food.

We first tested worms defective for TDC-1, the tyrosine decarboxylase enzyme required for synthesis of tyramine and its downstream product, octopamine. We found that $t d c-1$ mutants exhibited increased fasting quiescence, increased dwelling, and reduced roaming relative to wild type (Fig. $7 a$ ). These characteristics of fasting $t d c-1$ mutants largely overlap with those of feeding N2 worms (Fig. 3j), indicating that animals lacking tyramine and octopamine behave as if feeding even in the absence of food.

Loss of TDC-1 abolishes the worm's ability to produce both tyramine and octopamine. To determine whether lack of tyramine or octopamine was responsible for the altered behavior in $t d c-1$ mutants, we tested behavior after either exogenous tyramine or octopamine was applied. Addition of exogenous octopamine suppressed the increased fasting quiescence in $t d c-1$ mutants as well as in wild type. By contrast, exogenous tyramine slightly increased fasting quiescence in wild type, but not in $t d c-1$ mutants (Fig. 7b). These results suggest that octopamine is required for wild-type levels of fasting quiescence, and that octopamine acts to suppress quiescence.

We also found that treatment of both wild-type and $t d c-1$ worms with tyramine resulted in increased dwelling and reduced roaming, whereas octopamine treatment slightly reduced dwelling and slightly increased roaming (Fig. $7 c, d$ ). These results indicate that octopamine and tyramine have largely opposite roles for locomotion, with tyramine promoting reduced locomotion typical of feeding worms and octopamine promoting increased locomotion typical of fasting worms.

\section{Octopamine signaling is required for worms to appropriately} adapt behavior to fasting

Next, we examined the behavior of tbh-1 mutants, which lack only octopamine. We found that $t b h-1$ mutants, like $t d c-1$ mutants, exhibited elevated quiescence, elevated dwelling, and decreased roaming under fasting conditions compared with wild-type animals. The defects in quiescence and roaming, but not dwelling, were reversed by exogenous treatment with octopamine (Fig. $8 a, c-e$ ). These re- 

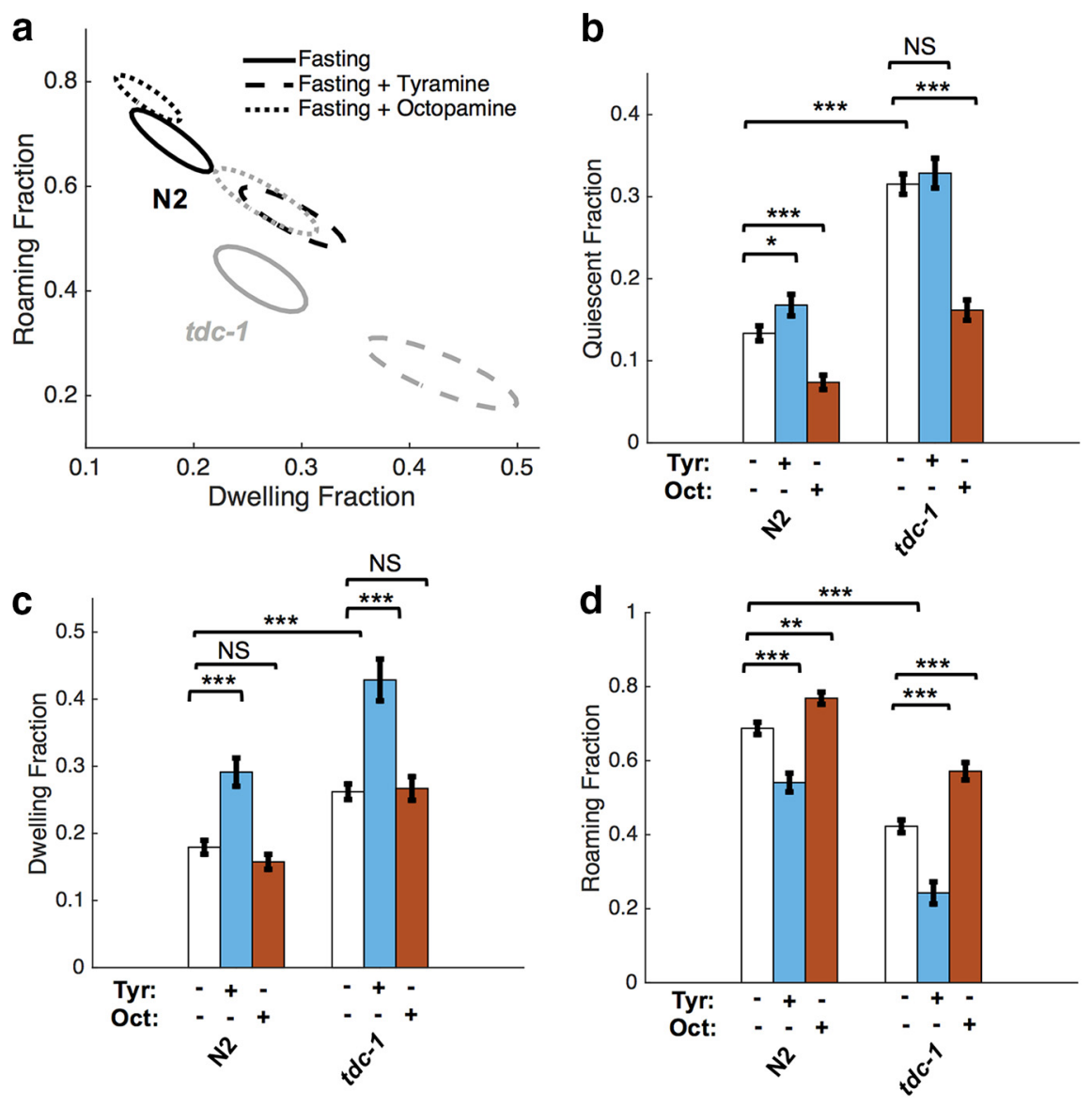

Figure 7. Octopamine and tyramine exert opposing effects on locomotory behavior. $\boldsymbol{a}$, Principal component ellipses illustrating fraction of time spent roaming versus dwelling for $\mathrm{N} 2$ (black) and tdc-1 (gray) under fasting (solid ellipses), fasting $+10 \mathrm{~mm}$ tyramine (dashed ellipses), or fasting $+10 \mathrm{~mm}$ octopamine (dotted ellipses) conditions. $\boldsymbol{b}$, Fraction of time spent quiescent. c, Fraction of time spent dwelling. Number of animals tested: N2 control $(n=103)$, N2 with tyramine $(n=52)$, N2 with octopamine $(n=60)$, tdc- 1 control $(n=104)$, tdc- 1 with tyramine $(n=52)$, tdc- 1 with octopamine $(n=60) . \boldsymbol{d}$, Fraction of time spent roaming. ${ }^{*} p<0.05 ;{ }^{* *} p<0.01 ;{ }^{* * *} p<0.005$.

sults show that in worms, as in flies, octopamine is responsible for the hyperactivity observed during fasting (Yang et al., 2015).

The increased dwelling, decreased roaming, and increased quiescence evident in fasting $t b h-1$ worms again recalls, like that of fasting $t d c-1$ worms, the behavior of feeding wild-type animals (Fig. $3 a, c, j, k$ ). The finding that fasting $t b h-1$ worms resemble feeding N2 worms suggests that octopamine, but not tyramine, is required for worms to adopt locomotion behaviors associated with fasting.

We next considered the behavior of $t b h-1$ mutants in the presence of food. Treatment of $t b h-1$ mutants with food caused a reduction in roaming and an increase in dwelling compared with fasting $t b h-1$ worms. This trend is similar to that observed in wild-type worms but occurred to a greater extent in $t b h-1$ animals (Fig. $3 k-n$ ). Feeding $t b h-1$ worms exhibit greatly increased dwelling, greatly reduced roaming, and no change in quiescence compared with both fasting $t b h-1$ worms. Feeding $t b h-1$ worms also exhibit more dwelling and less roaming than feeding wild-type worms, suggesting that these animals overcompensate their locomotion behavior to food compared with wild type.

The finding that feeding $t b h-1$ worms exhibit a large fraction of dwelling is consistent with a qualitative view of their behavior, which shows a fairly homogenous, low level of activity throughout the $16 \mathrm{~h}$ of the assay, compared with the relatively more heterogeneous behavior of both feeding wild-type and tph-1 worms
(Fig. $3 a-c, e, g, i)$. These results reinforce the notion that octopamine acts to promote movement, but also implies that this pathway acts during feeding as well as fasting.

Both SER-3 and SER-6 are required for exogenous octopamine's effects on locomotion

We next sought to determine the receptor(s) through which exogenous octopamine acts to excite roaming and suppress quiescence. We tested mutants for the three known octopamine receptors (Komuniecki et al., 2004, 2012; Sun et al., 2011). We found that response to octopamine was abolished in both ser- 3 and ser- 6 mutants but remained largely intact in octr-1 mutants (Fig. $8 a-e$ ). Treatment with exogenous octopamine significantly increased roaming and decreased quiescence in wild-type and octr-1 mutants, but was unable to do so in either ser- 3 or ser- 6 mutants. These results indicate that octopamine requires both SER-3 and SER-6 receptors to affect locomotion.

\section{SER-3 and SER-6 act in the SIA neurons to mediate octopamine's effect on locomotion}

We next sought to determine where the SER-3 and SER-6 receptors acted to mediate octopamine's effect on behavior. A previous report showed that SER-3 and SER-6 acted in the SIA neurons to mediate the transcription of the starvation-responsive transcription factor, the cAMP response element-binding protein (CREB; Yoshida et al., 2014). We therefore tested whether rescuing SER-3 and SER-6 in the SIA neurons was sufficient to restore wild-type response to octopamine.

We found that expressing SER-3 or SER-6 under the Pceh-17 promoter, which drives expression specifically in the SIA and ALA neurons, was sufficient to restore wild-type response to octopamine in ser-3 and ser- 6 mutants, respectively (Fig. $9 a-c$ ). A previous study found that octopamine modulated CREB levels in the SIA neurons, suggesting that SER-3 and SER-6 function in the SIA neurons and not in the ALA neurons (Suo et al., 2006; Yoshida et al., 2014). Therefore, our results suggest that SER-3 and SER-6 also function in the SIA neurons to mediate octopamine's effect on locomotion.

\section{Discussion}

Quantitative, high-throughput imaging enables characterization of complicated behavioral phenotypes

In this study, we used the WorMotel, a multi-well device designed for long-term behavioral imaging of $C$. elegans, to elucidate the molecular and neural pathways underlying the locomotion behavioral effects of the biogenic amines 5-HT and octopamine. By acquiring high-throughput behavioral measurements, we quantified the time spent quiescent, dwelling, and roaming for 48 worms simultaneously over the first $16 \mathrm{~h}$ of young-adulthood. Automated behavioral analyses like those described here will enable quantification of complicated behavioral phenotypes and 

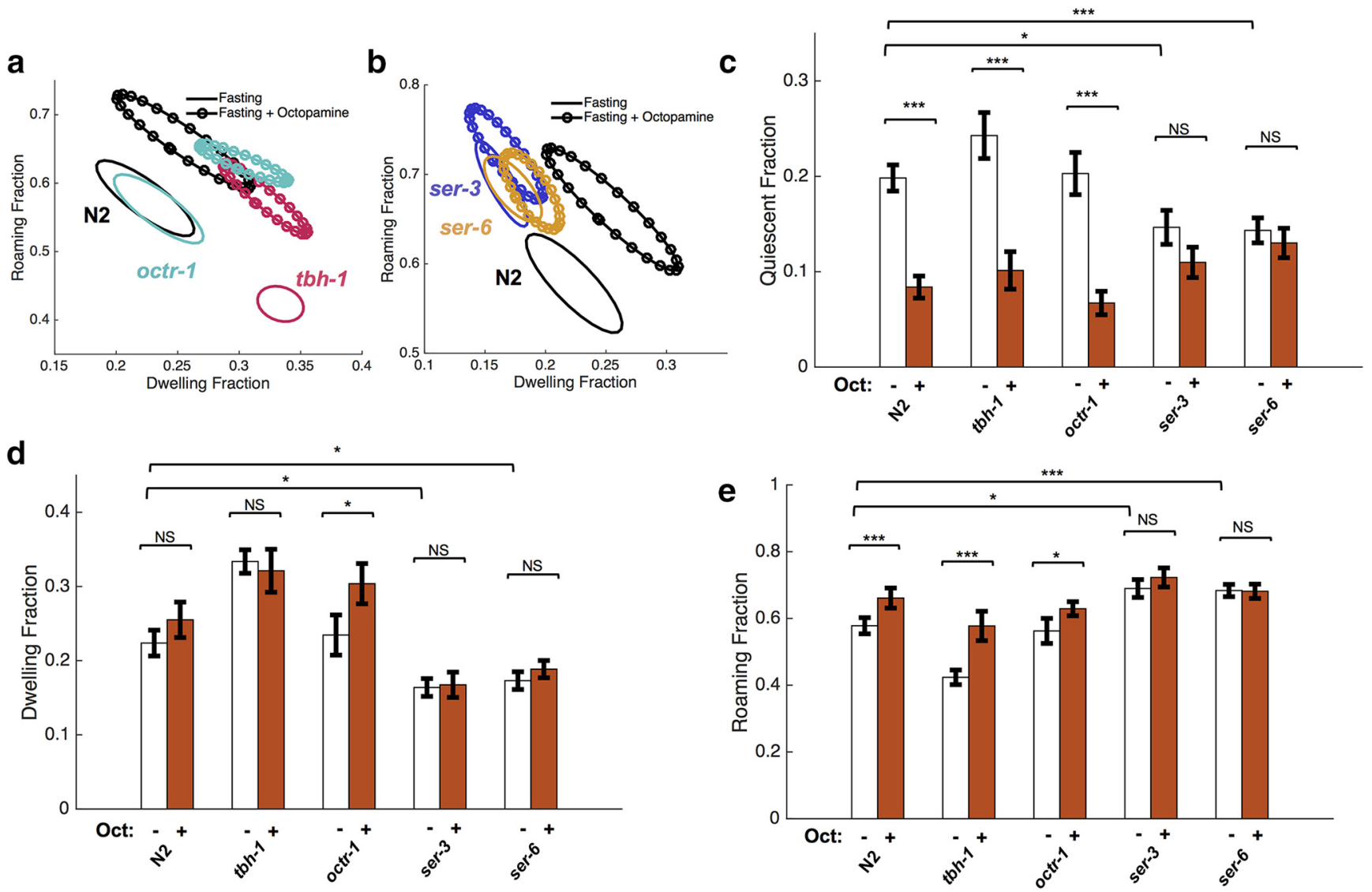

Figure 8. Exogenous octopamine promotes roaming and suppresses quiescence through both SER-6 and SER-3. $\boldsymbol{a}$, Principal component ellipses illustrating fraction of time spent roaming versus dwelling for N2 (black), tbh-1 (red), or octr-1 (teal) under fasting (solid ellipses) or fasting +10 mm octopamine (dotted ellipses) conditions. $\boldsymbol{b}$, Principal component ellipses illustrating fraction of time spent roaming versus dwelling for $\mathrm{N} 2$ (black), ser-3 (purple), and ser- 6 (orange) under fasting or fasting $+10 \mathrm{~mm}$ octopamine conditions. c, Fraction of time spent quiescent. Number of animals tested were equal for both with and without octopamine conditions: N2 $(n=80)$, tbh- $1(n=54)$, octr- $1(n=26)$, ser-3 $(n=56)$, ser-6 $(n=72)$. $\boldsymbol{d}$, Fraction of time spent dwelling.e, Fraction of time spent roaming. ${ }^{*} p<0.05 ;{ }^{* * *} p<0.005$.

a

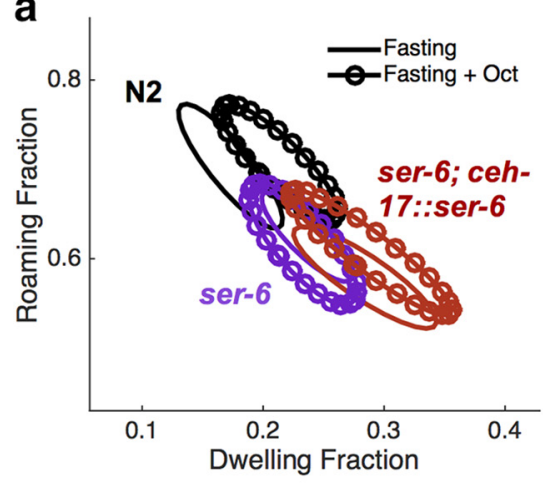

b

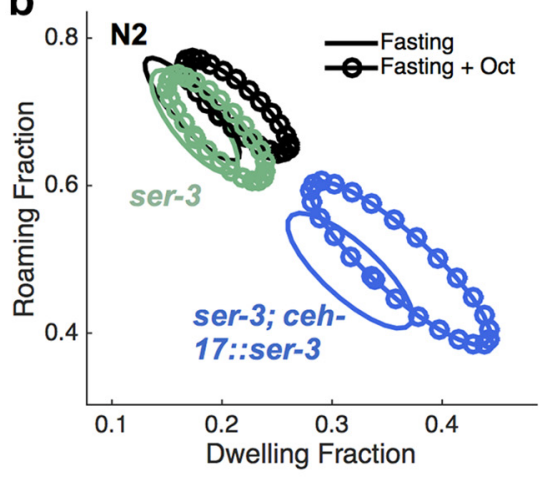

C

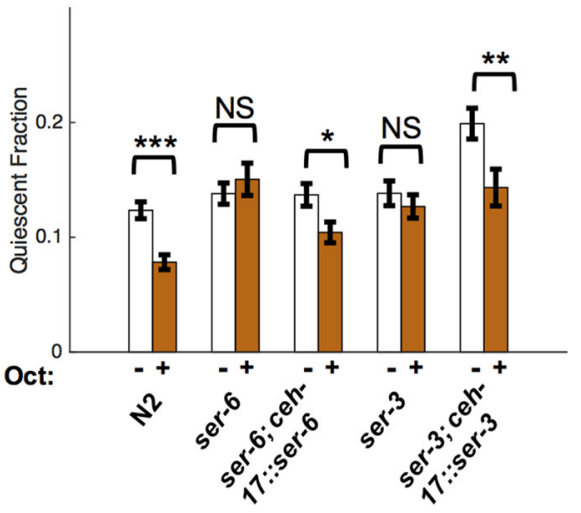

Figure 9. SER- 6 and SER-3 function in the SIA neurons to mediate octopamine's effects on behavior. $\boldsymbol{a}$, Principal component ellipses illustrating fraction of time spent roaming versus dwelling for N2 (black), ser-6 (purple), and ser-6; ceh-17::ser-6 (red) under fasting (solid ellipses) or fasting $+10 \mathrm{~mm}$ octopamine (dotted ellipses) conditions. b, Principal component ellipses illustrating fraction of time spent roaming versus dwelling for N2 (black), ser-3 (green), and ser-3;ceh-17::ser-3 (blue) under fasting or fasting +10 mm octopamine conditions. c, Fraction of time spent quiescent. Number of animals tested were equal for both with and without octopamine conditions: N2 ( $n=144)$, ser-6 $(n=48)$, ser-6; ceh-17::ser-6 $(n=48)$, ser-3 ( $n=96)$, ser-3; ceh-17::ser-3 $(n=64) .{ }^{*} p<0.05 ;{ }^{* *} p<0.01 ;{ }^{* * *} p<0.005$.

thus allow researchers to sort out how neural, genetic, and environmental variables act together to generate behavior.

As evidenced here and described previously, animals use the same basic behaviors under different experimental conditions. In C. elegans, these basic behaviors are quiescence, roaming, and dwelling. However, the probability of each respective behavior is modulated as necessary to achieve an organism's goals appropriate to the current environmental state. Therefore, the ability to automatically detect and annotate different behavioral phenotypes in a quantitative way is crucial for us to understand the nuanced way the relative weights of different behavioral states are modulated in response to the environment. 


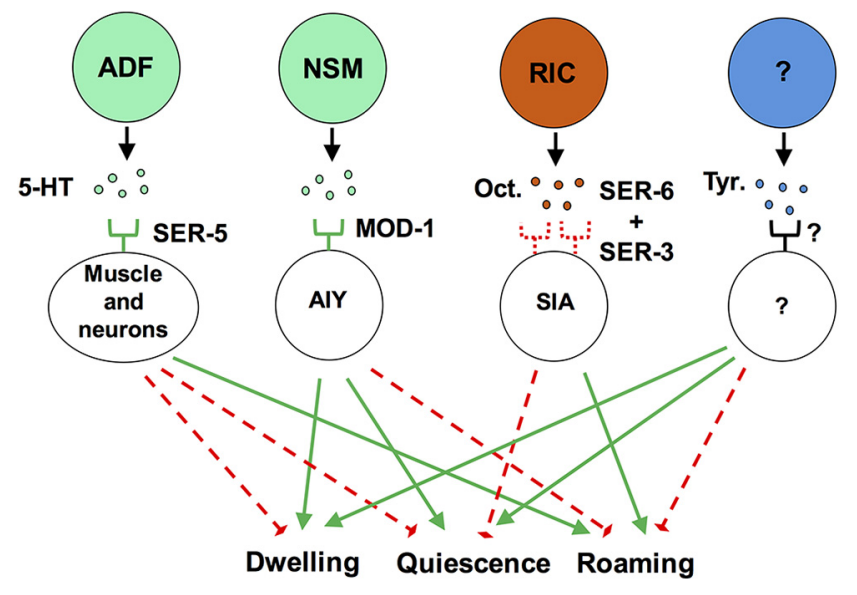

Figure 10. Model for biogenic amine locomotion effects.

Our findings support the prevailing view that 5-HT and octopamine signaling are required for appropriately adapting behavior to the presence and absence of food, respectively. We also describe the receptors and sites of action of these signaling molecules. Together, our results delineate the neural circuit and molecular mechanisms that appropriately govern locomotion behavior in relation to food levels (Fig. 10).

\section{Two 5-HT-producing neurons exert opposing effects on locomotion}

Our results suggest that ADF-produced 5-HT acts via SER-5 to promote roaming, whereas NSM-produced 5-HT acts via MOD-1 to promote dwelling. Therefore, serotonin acts both to increase and decrease locomotion, depending on its source. The fact that serotonin alone can both excite and suppress locomotion is supported by the fact that fasting $t b h-1$ mutants, which lack the roaming-promoting signal octopamine, are able to generate periods of both dwelling and roaming (Fig. $3 c, h$ ). This variation in behavior could be produced by the alternate release of 5-HT from ADF and NSM to produce periods of high and low locomotion, respectively.

\section{5-HT acts antagonistically through SER-5 and MOD-1 to regulate locomotion}

Previous results have shown that other 5-HT receptors act to slow locomotion in C. elegans (Ranganathan et al., 2000). Our result suggests that SER-5 is the sole receptor through which 5-HT can stimulate roaming. Similarly, MOD-1 appears to be the primary receptor through which 5-HT promotes dwelling (Fig. 5). Therefore, we confirm that 5-HT promotes dwelling via MOD-1 and show for the first time that 5-HT promotes roaming via SER-5 (Fig. 10).

Our results suggest that $\mathrm{ADF}$ is an activity-promoting neuron and NSM is activity-suppressing neuron. Therefore, it is plausible that ADF-produced 5-HT acts via SER-5 to regulate locomotion, as previously found in the context of copper-avoidance behavior (Guo et al., 2015). Our results would then suggest that NSM-produced 5-HT therefore acts via MOD-1 to suppress locomotion.

MOD-1 acts in the AIY neurons and SER-5 acts in muscles and neurons to regulate locomotion

We found that rescuing MOD-1 under either its endogenous promoter or the TTX-3 promoter restored wild-type response to 5-HT. Previous reports have also found that expressing MOD-1 under the TTX-3 promoter, which is strongly expressed in the
AIY neurons, is required to promote dwelling in feeding wildtype worms (Hapiak et al., 2009; Flavell et al., 2013). We confirm this role for promotion of dwelling by MOD-1 in the AIY neurons. Given that MOD-1 is a 5-HT-gated chloride channel (Ranganathan et al., 2000) and optogenetic activation of AIY promotes roaming (Flavell et al., 2013), it is likely that MOD-1 acts to silence AIY in response to 5-HT. Therefore, MOD-1 likely acts to suppress activity in the AIY neurons to promote dwelling in response to 5-HT.

We found that SER-5 could act in either muscle or neurons to promote movement in response to 5 -HT. A previous study found that SER-5 acted in vulval muscle to regulate serotonergic stimulation of egg laying (Hapiak et al., 2009). However, this study did not describe a role for SER-5 in regulating movement. Another study found that SER-5 acts in the nervous system specifically in the ASH neurons to mediate response times to octanol (Harris et al., 2009). Therefore, SER-5 occupies multiple tissue sites of action to mediate diverse behaviors. Egg laying is associated with a transient increase in movement (Waggoner et al., 2000; McCloskey et al., 2017). Therefore, it is possible that SER-5 acts in the muscle to increase egg-laying rate, which secondarily acts to increase movement. An alternative possibility is that SER-5 acts directly on body wall muscles to promote movement. We can, however, rule out a role for the ASH neuron in regulating locomotion behavior in response to 5-HT (Fig. $6 f-j$ ). More work is required to identify whether SER-5 acts endogenously in neurons, muscles, or both to regulate locomotion behavior.

\section{Octopamine promotes roaming behavior via SER-3 and SER-6 in the SIA neurons}

Both fasting and treatment with exogenous octopamine promote CREB transcription factor activity in the SIA neurons (Suo et al., 2006; Yoshida et al., 2014). This effect requires the presence of both SER-3 and SER-6. Therefore, it has been hypothesized that these receptors act nonredundantly in the SIA neurons to mediate octopamine's effects in response to starvation, perhaps via a threshold effect or dimerization.

We found that SER-3 and SER-6 also mediate octopamine's effect on behavior, and that both receptors function in SIA to mediate this effect. Therefore, our results further support the notion that both SER-3 and SER-6 are required for octopamine's effect on the response to fasting by modulating quiescent behavior (Figs. 8, 9). Our results suggest that the CREB transcription factor may play important roles in regulating fasting locomotion behavior.

\section{5-HT and octopamine promote behaviors associated with feeding and fasting, respectively}

Our results suggest that food initiates release of 5-HT, but that in wild-type animals a reserve of octopamine signaling antagonizes this food-induced 5-HT release to antagonize the effects of 5-HT on behavior and promote roaming even when food is present. This explains why the behavior of feeding N2 worms is different from the behavior of $\mathrm{N} 2$ worms treated with exogenous 5-HT. The dose of exogenous 5-HT permeating the cuticle in these experiments may be much larger than that released endogenously, so exogenously supplied 5-HT dominates the endogenous octopamine signal that normally counteracts 5-HT's effect. By contrast, no octopamine is present to counteract the 5-HT released in tbh-1 mutants supplied with food, so the behavior of these animals resembles that observed in wild type treated with exogenous 5-HT (Figs. 3k, 7a,b)

Despite the finding that 5-HT treatment primarily promotes feeding-associated locomotion phenotypes and octopamine treat- 
ment primarily promotes fasting-associated phenotypes, we find that both transmitters are required for wild-type behavior under both feeding and fasting conditions. We find that although well fed wild-type animals mostly make small movements, they also exhibit periods of high locomotion activity. This is consistent with previously published reports showing that on food, worms spend time primarily in dwelling state but also exhibit short-lived roaming states (Flavell et al., 2013).

Our results suggest that by continuing to release lower levels of the nondominant biogenic amine-octopamine under feeding conditions and 5-HT under fasting conditions-worms continue to maintain access to a variety of behaviors, some of which normally associated with an environmental state they do not currently find themselves in. By reducing, but not eliminating, behaviors associated with environmental conditions not currently being experienced, animals maintain a high degree of adaptability.

\section{Conserved roles of 5-HT and octopamine}

5-HT and octopamine have conserved roles over diverse phyla. 5-HT is found in the protozoan Tetrahymena as an intracellular regulator of the cAMP pathway (Turlejski, 1996), a known regulator of behavior. The majority of vertebrate 5-HT receptor families also modulate cAMP levels through their effects on adenylate cyclase, indicating a remarkable degree of conservation of the molecular role of this signaling molecule over 2 billion years of evolution (Turlejski, 1996). The evolutionary conservation of these receptors' downstream signaling pathways suggests that understanding the molecular roles of these receptors in model organisms may contribute to our understanding of these receptors' roles in mammals.

Octopamine is found primarily in invertebrates, but also in trace amounts in mammals, and is structurally similar to the fightor-flight hormones adrenaline and noradrenaline. Here we find that octopamine's role in worms is similar to that in flies, where it induces hyperactivity upon fasting as well as wake-promotion (Crocker and Sehgal, 2008). However, it was not previously known which receptors octopamine acted through to induce hyperactivity in Drosophila. The conservation of behavioral effects of biogenic amines suggests that roles for specific receptors may also be preserved.

Understanding the role of these signals in invertebrates may inform our understanding of the molecular bases for more complex manifestations of heightened activity based on these signals in mammals, such as aggression and anxiety.

\section{References}

Alkema MJ, Hunter-Ensor M, Ringstad N, Horvitz HR (2005) Tyramine functions independently of octopamine in the Caenorhabditis elegans nervous system. Neuron 46:247-260. CrossRef Medline

Andrews MT (2007) Advances in molecular biology of hibernation in mammals. BioEssays 29:431-440. CrossRef Medline

Brenner S (1974) The Genetics of C. elegans. Genetics 77:71-94. Medline

Carey HV, Andrews MT, Martin SL (2003) Mammalian hibernation: cellular and molecular responses to depressed metabolism and low temperature. Physiol Rev 83:1153-1181. CrossRef Medline

Carre-Pierrat M, Baillie D, Johnsen R, Hyde R, Hart A, Granger L, Ségalat L (2006) Characterization of the Caenorhabditis elegans G-protein-coupled serotonin receptors. Invert Neurosci 6:189-205. CrossRef Medline

Chase DL, Koelle MR 2007 Biogenic amine neurotransmitters in C. elegans. In: WormBook (The C. elegans Research Community, eds). Available at http://www.wormbook.org. CrossRef

Churgin MA, Fang-Yen C (2015) An imaging system for C. elegans behavior. Methods Mol Biol 1327:199-207. CrossRef Medline

Churgin MA, Jung S-K, Yu C-C, Chen X, Raizen D, Fang-Yen C (2017) Longitudinal imaging of C. elegans with a microfabricated device reveals variation in behavioral decline during aging. eLife 6:e26652. CrossRef Medline

Crocker A, Sehgal A (2008) Octopamine regulates sleep in Drosophila through protein kinase A-dependent mechanisms. J Neurosci 28:93779385. CrossRef Medline

Cunningham KA, Hua Z, Srinivasan S, Liu J, Lee BH, Edwards RH, Ashrafi K (2012) AMP-activated kinase links serotonergic signaling to glutamate release for regulation of feeding behavior in C. elegans. Cell Metab 16:113121. CrossRef Medline

Curran KP, Chalasani SH (2012) Serotonin circuits and anxiety: What can invertebrates teach us? Invert Neurosci 12:81-92. Medline

Davis MW, Somerville D, Lee RY, Lockery S, Avery L, Fambrough DM (1995) Mutations in the Caenorhabditis elegans Na, K-ATPase gene, alpha-subunit gene, eat-6, disrupt excitable cell function. J Neurosci 15: 8408-8418. Medline

Estevez AO, Cowie RH, Gardner KL, Estevez M (2006) Both insulin and calcium channel signaling are required for developmental regulation of serotonin synthesis in the chemosensory ADF neurons of Caenorhabditis elegans. Dev Biol 298:32-44. CrossRef Medline

Flavell SW, Pokala N, Macosko EZ, Albrecht DR, Larsch J, Bargmann CI (2013) Serotonin and the neuropeptide PDF initiate and extend opposing behavioral states in C. elegans. Cell 154:1023-1035. CrossRef Medline

Gallagher T, Kim J, Oldenbroek M, Kerr R, You YJ (2013) ASI regulates satiety quiescence in C. elegans. J Neurosci 33:9716-9724. CrossRef Medline

Ghosh R, Emmons SW (2008) Episodic swimming behavior in the nematode C. elegans. J Exp Biol 211:3703-3711. CrossRef Medline

Guo M, Wu TH, Song YX, Ge MH, Su CM, Niu WP, Li LL, Xu ZJ, Ge CL, Al-Mhanawi MT, Wu SP, Wu ZX (2015) Reciprocal inhibition between sensory ASH and ASI neurons modulates nociception and avoidance in Caenorhabditis elegans. Nat Commun 6:5655. CrossRef Medline

Gürel G, Gustafson MA, Pepper JS, Robert Horvitz HR, Koelle MR (2012) Receptors and other signaling proteins required for serotonin control of locomotion in Caenorhabditis elegans. Genetics 192:1359-1371. CrossRef Medline

Hapiak VM, Hobson RJ, Hughes L, Smith K, Harris G, Condon C, Komuniecki P, Komuniecki RW (2009) Dual excitatory and inhibitory serotonergic inputs modulate egg laying in Caenorhabditis elegans. Genetics 181:153-63. CrossRef Medline

Harris GP, Hapiak VM, Wragg RT, Miller SB, Hughes LJ, Hobson RJ, Steven R, Bamber B, Komuniecki RW (2009) Three distinct amine receptors operating at different levels within the locomotory circuit are each essential for the serotonergic modulation of chemosensation in Caenorhabditis elegans. J Neurosci 29:1446-1456. CrossRef Medline

Hart A (2006) Behavior. In: WormBook (The C. elegans Research Community, eds). Available at: http://www.wormbook.org CrossRef

Komuniecki RW, Hobson RJ, Rex EB, Hapiak VM, Komuniecki PR (2004) Biogenic amine receptors in parasitic nematodes: what can be learned from Caenorhabditis elegans? Mol Biochem Parasitol 137:1-11. CrossRef Medline

Komuniecki R, Harris G, Hapiak V, Wragg R, Bamber B (2012) Monoamines activate neuropeptide signaling cascades to modulate nociception in C. elegans: a useful model for the modulation of chronic pain? Invert Neurosci 12:53-61. CrossRef Medline

Lam RV (2006) Sleep disturbances and depression: a challenge for antidepressants. Int Clin Psychopharmacol 21(Suppl 1):S25-S29. CrossRef Medline

Liang B, Moussaif M, Kuan CJ, Gargus JJ, Sze JY (2006) Serotonin targets the DAF-16/FOXO signaling pathway to modulate stress responses. Cell Metab 4:429-440. CrossRef Medline

McCloskey RJ, Fouad AD, Churgin MA, Fang-Yen C (2017) Food responsiveness regulates episodic behavioral states in Caenorhabditis elegans. J Neurophysiol 117:1911-1934. CrossRef Medline

Mills H, Wragg R, Hapiak V, Castelletto M, Zahratka J, Harris G, Summers P, Korchnak A, Law W, Bamber B, Komuniecki R (2012) Monoamines and neuropeptides interact to inhibit aversive behaviour in Caenorhabditis elegans. EMBO J 31:667-678. CrossRef Medline

Nelson OL, Jansen HT, Galbreath E, Morgenstern K, Gehring JL, Rigano KS, Lee J, Gong J, Shaywitz AJ, Vella CA, Robbins CT, Corbit KC (2014) Grizzly bears exhibit augmented insulin sensitivity while obese prior to a reversible insulin resistance during hibernation. Cell Metab 20:376-382. CrossRef Medline

Noble T, Stieglitz J, Srinivasan S (2013) An integrated serotonin and octo- 
pamine neuronal circuit directs the release of an endocrine signal to control C. elegans body fat. Cell Metab 18:672-684. CrossRef Medline

Ranganathan R, Cannon SC, Horvitz HR (2000) MOD-1 is a serotonin-gated chloride channel that modulates locomotory behaviour in C. elegans. Nature 408:470-475. CrossRef Medline

Rex E, Komuniecki RW (2002) Characterization of a tyramine receptor from Caenorhabditis elegans. J Neurochem 82:1352-1359. CrossRef Medline

Rex E, Molitor SC, Hapiak V, Xiao H, Henderson M, Komuniecki R (2004) Tyramine receptor (SER-2) isoforms are involved in the regulation of pharyngeal pumping and foraging behavior in Caenorhabditis elegans. J Neurochem 91:1104-1115. CrossRef Medline

Roeder T (2005) Tyramine and octopamine: ruling behavior and metabolism. Annu Rev Entomol 50:447-477. CrossRef Medline

Sawin ER, Ranganathan R, Horvitz HR (2000) C. elegans locomotory rate is modulated by the environment through a dopaminergic pathway and by experience through a serotonergic pathway. Neuron 26:619-631. CrossRef Medline

Ségalat L, Elkes DA, Kaplan JM (1995) Modulation of serotonin-controlled behaviors by Go in Caenorhabditis elegans. Science 267:1648-1651. CrossRef Medline

Sengupta P (2013) The belly rules the nose: feeding state-dependent modulation of peripheral chemosensory responses. Curr Opin Neurobiol 23: 68-75. CrossRef Medline

Shepherd RF, Ilievski F, Choi W, Morin SA, Stokes AA, Mazzeo AD, Chen X, Wang M, Whitesides GM (2011) Multigait soft robot. Proc Natl Acad Sci U S A 108:20400-20403. CrossRef Medline

Song BM, Faumont S, Lockery S, Avery L (2013) Recognition of familiar food activates feeding via an endocrine serotonin signal in Caenorhabditis elegans. eLife 2:e00329. CrossRef Medline

Stiernagle T (2006) Maintenance of C. Elegans. In: WormBook (The C. elegans Research Community, eds). Available at: http://www.wormbook.org. CrossRef

Sun J, Singh V, Kajino-Sakamoto R, Aballay A (2011) Neuronal GPCR controls innate immunity by regulating noncanonical unfolded protein response genes. Science 332:729-732. CrossRef Medline

Suo S, Kimura Y, Van Tol HH (2006) Starvation induces cAMP response element-binding protein-dependent gene expression through octopamine- $\mathrm{G}_{\mathrm{q}}$ signaling in Caenorhabditis elegans. J Neurosci 26:10082-10090. CrossRef Medline

Sze JY, Victor M, Loer C, Shi Y, Ruvkun G (2000) Food and metabolic signalling defects in a Caenorhabditis elegans serotonin-synthesis mutant. Nature 403:560-564. CrossRef Medline

Sze JY, Zhang S, Li J, Ruvkun G (2002) The C. elegans POU-domain transcription factor UNC-86 regulates the tph-1 tryptophan hydroxylase gene and neurite outgrowth in specific serotonergic neurons. Development 129:3901-3911. Medline

Tecott LH (2007) Serotonin and the orchestration of energy balance. Cell Metab 6:352-361. CrossRef Medline

Turlejski K (1996) Evolutionary ancient roles of serotonin: long-lasting regulation of activity and development. Acta Neurobiol Exp (Wars) 56:619636. Medline

van Breukelen F, Martin SL (2015) The hibernation continuum: physiological and molecular aspects of metabolic plasticity in mammals. Physiology 30:273-281. CrossRef Medline

Waggoner LE, Zhou GT, Schafer RW, Schafer WR (1998) Control of alternative behavioral states by serotonin in Caenorhabditis elegans. Neuron 21:203-214. CrossRef Medline

Waggoner LE, Hardaker LA, Golik S, Schafer WR (2000) Effect of a neuropeptide gene on behavioral states in C. elegans egg-laying. Genetics 154: 1181-1192. Medline

Wragg RT, Hapiak V, Miller SB, Harris GP, Gray J, Komuniecki PR, Komuniecki RW (2007) Tyramine and octopamine independently inhibit serotoninstimulated aversive behaviors in Caenorhabditis elegans through two novel amine receptors. J Neurosci 27:13402-13412. CrossRef Medline

Yang Z, Yu Y, Zhang V, Tian Y, Qi W, Wang L (2015) Octopamine mediates starvation-induced hyperactivity in adult Drosophila. Proc Natl Acad Sci U S A 112:5219-5224. CrossRef Medline

Yoshida M, Oami E, Wang M, Ishiura S, Suo S (2014) Nonredundant function of two highly homologous octopamine receptors in food-deprivationmediated signaling in Caenorhabditis elegans. J Neurosci Res 92:671-678. CrossRef Medline

You YJ, Kim J, Raizen DM, Avery L (2008) Insulin, cGMP, and TGF- $\beta$ signals regulate food intake and quiescence in C. elegans: a model for satiety. Cell Metab 7:249-257. CrossRef Medline

Zhang Y, Lu H, Bargmann CI (2005) Pathogenic bacteria induce aversive olfactory learning in Caenorhabditis elegans. Nature 438:179-184. CrossRef Medline

Zheng X, Chung S, Tanabe T, Sze JY (2005) Cell-type specific regulation of serotonergic identity by the C. elegans LIM-homeodomain factor LIM-4. Dev Biol 286:618-628. CrossRef Medline 